International Journal of Pure and Applied Mathematics

Volume 84 No. 5 2013, 525-537

ISSN: 1311-8080 (printed version); ISSN: 1314-3395 (on-line version)

url: http://www.ijpam.eu

doi: http://dx.doi.org/10.12732/ijpam.v84i5.6

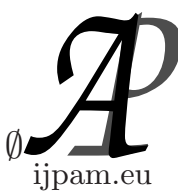

\title{
SEMIGROUP OF INJECTIVE PARTIAL TRANSFORMATIONS WITH BOUNDED GAP AND FIXED DEFECT
}

\author{
Boorapa Singha \\ School of Mathematics and Statistics \\ Faculty of Science and Technology \\ Chiangmai Rajabhat University \\ Chiangmai, 50300, THAILAND
}

\begin{abstract}
Let $X$ be an infinite set and suppose that $\aleph_{0} \leq q \leq|X|$. In 2004, Pinto and Sullivan considered algebraic properties of $P S(q)$, the partial Baer-Levi semigroup consisting of all injective partial transformations $\alpha$ of $X$ such that $|X \backslash X \alpha|=q$. They also determined its subsemigroup $S(q, r)=\{\alpha \in$ $P S(q):|X \backslash \operatorname{dom} \alpha| \leq r\}$ where $\aleph_{0} \leq r \leq|X|$. Recently, Singha and Sanwong showed that, when $q<|X|$, almost every maximal subsemigroup of $P S(q)$ is induced by a maximal subsemigroup of $S(q, r)$. Here, we use their work to describe some algebraic properties of $S(q, r)$ including its Green's relation and its ideal structure.
\end{abstract}

AMS Subject Classification: 20M20

Key Words: injective partial transformation, partial Baer-Levi semigroup, gap, defect

\section{Introduction}

Suppose that $X$ is a non-empty set, and let $I(X)$ denote the symmetric inverse semigroup on $X$ (see [1] vol 1, p 29): that is, the set of all injective mappings from $X$ into $X$. For any $\alpha \in I(X)$, we let $\operatorname{dom} \alpha$ and $\operatorname{ran} \alpha$ (or $X \alpha$ ) denote the

Received: November 15, 2012

(c) 2013 Academic Publications, Ltd. url: www.acadpubl.eu 
domain of $\alpha$ and range of $\alpha$, respectively. We also write

$$
g(\alpha)=|X \backslash \operatorname{dom} \alpha|, \quad d(\alpha)=|X \backslash \operatorname{ran} \alpha|, \quad r(\alpha)=|\operatorname{ran} \alpha|,
$$

and refer to these cardinals as the gap, the defect and the rank of $\alpha$, respectively. For an infinite cardinal $q$ such that $\aleph_{0} \leq q \leq|X|$, we write

$$
P S(q)=\{\alpha \in I(X): d(\alpha)=q\}
$$

where $P S(q)$ is the partial Baer-Levi semigroup on $X$. In [3] Theorem 1 , Pinto and Sullivan showed that $P S(q)$ is right reductive in the sense that, if $\alpha \gamma=\beta \gamma$ for all $\gamma \in P S(q)$ implies $\alpha=\beta(\alpha, \beta \in P S(q))$, and left reductive which defined dually (see [1] vol 1, p 9), and they characterised the Green's relations and ideals of $P S(q)$. They also determined its subsemigroups

$$
S_{r}=\{\alpha \in I(X): d(\alpha)=q \text { and } g(\alpha) \leq r\}
$$

where $\aleph_{0} \leq r \leq|X|$. In fact, when $r=|X|$ we have $S_{r}=P S(q)$, then we may regard $S_{r}$ as a generalization of $P S(q)$. More recently, in [4], Singha and Sanwong studied maximal subsemigroups of $P S(q)$. In particular, when $q<|X|$ they found that almost every maximal subsemigroup of $P S(q)$ is induced by a maximal subsemigroup of $S_{r}$ for some cardinal $r$ such that $q \leq r<|X|$ (see [4] Theorem 3.5).

In this paper, we examine a subsemigroup $S_{r}$ of $P S(q)$ and we write $S(q, r)$ in place of $S_{r}$ to highlight the defect $q$ and the maximum gap $r$ of elements in this semigroup.

\section{Basic Properties}

We modify the convention introduced in [1] vol 2, p 241: namely, if $\alpha \in I(X)$ is non-zero, then we write

$$
\alpha=\left(\begin{array}{l}
a_{i} \\
x_{i}
\end{array}\right)
$$

and take as understood that the subscript $i$ belongs to some (unmentioned) index set $I$, that the abbreviation $\left\{x_{i}\right\}$ denotes $\left\{x_{i}: i \in I\right\}$, and that $X \alpha=$ $\operatorname{ran} \alpha=\left\{x_{i}\right\}, x_{i} \alpha^{-1}=\left\{a_{i}\right\}$ and $\operatorname{dom} \alpha=\left\{a_{i}: i \in I\right\}$. For simplicity, if $A \subseteq X$, we sometimes write $A \alpha$ in place of $(A \cap \operatorname{dom} \alpha) \alpha$, and if $A \subseteq \operatorname{dom} \alpha$, then we write $\alpha \mid A$ to mean the restriction of $\alpha$ on $A$.

In this paper, $Y=A \dot{\cup} B$ means $Y$ is a disjoint union of $A$ and $B$. Also, for each non-empty $A \subseteq X$, we write $\operatorname{id}_{A}$ for the identity transformation on $A$ : 
these mappings constitute all the idempotents in $I(X)$ and it is known from [3] that $\operatorname{id}_{A} \in P S(q)$ precisely when $|X \backslash A|=q$. As usual, $\emptyset$ denotes the empty (one-to-one) mapping which acts as a zero for $I(X)$ and in [3] Theorem 1, the authors showed that it belongs to $P S(q)$ precisely when $q=|X|$.

Theorem 1. Suppose that $|X| \geq q \geq \aleph_{0}$ and $|X| \geq r \geq \aleph_{0}$. Then $S(q, r)$ contains a zero precisely when $|X|=q=r$. Moreover, $S(q, r)$ is a right and left reductive semigroup without identity.

Proof. Suppose that $\gamma$ is a zero for $S(q, r)$. For each $x \in X$, if $x \in X \gamma$, then we write $X=P \dot{\cup} Q \dot{\cup}\{x\}$ where $|P|=|X|,|Q|=q$. Then a bijection $\beta: X \rightarrow P$ belongs to $S(q, r)$. Since $\gamma$ is a zero for $S(q, r)$, we have $\gamma=\gamma \beta$. Then $X \gamma \subseteq X \beta=P$. This follows that $Q \dot{\cup}\{x\}=X \backslash P \subseteq X \backslash X \gamma$, a contradiction. This means that $\gamma=\emptyset$. Since $d(\emptyset)=|X|=g(\emptyset)$, then $\emptyset$ belongs to $S(q, r)$ precisely when $|X|=q=r$.

To show that $S(q, r)$ is right reductive, suppose that $\alpha, \beta \in S(q, r)$ and $\alpha \gamma=\beta \gamma$ for all $\gamma \in S(q, r)$. If (say) $\alpha=\emptyset$, then $|X|=q=r$. In this case $\operatorname{id}_{X \beta} \in S(q, r)$ and thus $\beta=\beta \cdot \operatorname{id}_{X \beta}=\alpha . \operatorname{id}_{X \beta}=\emptyset$. Now suppose $\alpha, \beta \neq \emptyset$ and we will consider in two cases. First, if $q \leq r$, then $\operatorname{id}_{X \alpha} \in S(q, r)$ and thus $\alpha=\alpha . \operatorname{id}_{X \alpha}=\beta \cdot \operatorname{id}_{X \alpha}$. This implies $\operatorname{dom} \alpha \subseteq \operatorname{dom} \beta$ and $\beta \mid \operatorname{dom} \alpha=\alpha$. Similarly, we have $\beta=\beta \cdot$ id $_{X \beta}=\alpha \cdot$ id $_{X \beta}$ since $\operatorname{id}_{X \beta} \in S(q, r)$, and hence $\operatorname{dom} \beta \subseteq \operatorname{dom} \alpha$. It follows that $\alpha=\beta$. If $r<q$, then we write $X \backslash X \alpha=Q_{1} \cup \dot{\cup} Q_{2} \cup \dot{\cup} R$ where $\left|Q_{1}\right|=q=\left|Q_{2}\right|,|R|=r$. Define

$$
\lambda=\left(\begin{array}{cc}
X \alpha & Q_{1} \cup Q_{2} \\
X \alpha & Q_{1}
\end{array}\right) \in S(q, r)
$$

where $\lambda \mid X \alpha=\operatorname{id}_{X \alpha}$ and $\lambda: Q_{1} \cup Q_{2} \rightarrow Q_{1}$ is a bijection. Then $\alpha=\alpha \lambda=\beta \lambda$. This implies that $\operatorname{dom} \alpha \subseteq \operatorname{dom} \beta$ and $\beta \mid \operatorname{dom} \alpha=\alpha$. Similarly, by using the same arguments we can show that $\operatorname{dom} \beta \subseteq \operatorname{dom} \alpha$ and this implies $\alpha=\beta$. Therefore, $S(q, r)$ is right reductive.

Now suppose that $\gamma \alpha=\gamma \beta$ for all $\gamma \in S(q, r)$. As before, if (say) $\alpha=\emptyset$, then $|X|=q=r$. In this case, $\operatorname{id}_{x} \in S(q, r)$ for all $x \in X$. Thus, $\emptyset=\operatorname{id}_{x} \cdot \alpha=\operatorname{id}_{x} \cdot \beta$ for all $x \in X$. This implies that $\beta=\emptyset$. Now we suppose that $\alpha, \beta \neq \emptyset$, then, for each $t \in \operatorname{dom} \alpha$, we write $X=P \cup \dot{\cup} Q \dot{\cup}\{t$ where $|P|=|X|,|Q|=q$. We define

$$
\mu=\left(\begin{array}{cc}
X \backslash\{t\} & t \\
P & t
\end{array}\right)
$$

where $\mu: X \backslash\{t\} \rightarrow P$ is a bijection. Then $\mu \in S(q, r)$ and $\mu \alpha=\mu \beta$. Therefore $t \mu \alpha=t \mu \beta$, and thus $t \alpha=t \beta$. Therefore $t \in \operatorname{dom} \beta$, that is, $\operatorname{dom} \alpha \subseteq \operatorname{dom} \beta$ and 
$\beta \mid \operatorname{dom} \alpha=\alpha$. Similarly, we can show that $\operatorname{dom} \beta \subseteq \operatorname{dom} \alpha$ and therefore $\alpha=\beta$, that is, $S(q, r)$ is left reductive.

Finally, to see that $S(q, r)$ has no identity. We observe that, for each $x \in X$, $x \in X \alpha$ for some $\alpha \in S(q, r)$. Suppose that $\gamma$ is the identity in $S(q, r)$. Then $\alpha \gamma=\alpha$. Thus, if $a \alpha=x$, then $x=a \alpha=a \alpha \gamma=x \gamma$. That is, $\gamma=\mathrm{id}_{X}$, and so $d(\gamma)=0$, contrary to $\gamma \in S(q, r)$. Therefore $S(q, r)$ has no identity.

Theorem 2. Suppose that $|X| \geq q \geq \aleph_{0}$ and $|X| \geq r \geq \aleph_{0}$. Then $S(q, r)$ contains idempotents precisely when $q \leq r$. In this case, the set of all idempotents in $S(q, r)$ is

$$
E(S(q, r))=\left\{\operatorname{id}_{A}: A \subseteq X,|X \backslash A|=q\right\} .
$$

Proof. Suppose that $\alpha$ is an idempotent in $S(q, r)$. If $\alpha=\emptyset$, then $|X|=$ $q=r$ and $\alpha$ is a one-to-one mapping on the empty set, and we have that $|X \backslash \emptyset|=q$. Suppose that $\alpha \neq \emptyset$ and let $x \in \operatorname{dom} \alpha$. Then $x \alpha=x \alpha^{2}$, and thus $x \alpha=x$ since $\alpha$ is injective. Therefore $\alpha=\operatorname{id}_{A}$ for some subset $A$ of $X$. Moreover, since $g(\alpha) \leq r$ and $d(\alpha)=q$, we have $q=|X \backslash A| \leq r$. Conversely, suppose $q \leq r$. Then for any subset $Q$ of $X$ with $|Q|=q$, we see that $\alpha=\operatorname{id}_{X \backslash Q}$ is an idempotent, and it belongs to $S(q, r)$ since $d(\alpha)=g(\alpha)=|Q|=q \leq r$.

\section{Regular Elements}

In [3] Corollary 1, the authors showed that

$$
R(q)=\{\alpha \in I(X): g(\alpha)=q=d(\alpha)\}
$$

is the largest regular subsemigroup of $P S(q)$. Moreover, $R(q)$ is an inverse semigroup. Now we characterise all regular elements in $S(q, r)$.

Theorem 3. Suppose that $|X| \geq q \geq \aleph_{0}$ and $|X| \geq r \geq \aleph_{0}$. Then $S(q, r)$ contains regular elements precisely when $q \leq r$. In this case, $\alpha$ is regular in $S(q, r)$ if and only if $g(\alpha)=q$.

Proof. Let $\alpha \in S(q, r)$. If $\alpha$ is a regular element in $S(q, r)$, then $\alpha=\alpha \beta \alpha$ for some $\beta \in S(q, r)$. For convenience, we say

$$
\alpha=\left(\begin{array}{l}
a_{i} \\
x_{i}
\end{array}\right),
$$

then $a_{i} \alpha \beta \alpha=a_{i} \alpha$, thus $a_{i} \alpha \beta=a_{i}$ (since $\alpha$ is injective) and it follows that $x_{i} \beta=a_{i}$. That is, $X \alpha \subseteq \operatorname{dom} \beta$ and

$$
X \alpha \beta=(X \alpha \cap \operatorname{dom} \beta) \beta=\left\{x_{i}\right\} \beta=\left\{a_{i}\right\}=\operatorname{dom} \alpha .
$$


Therefore, since $\alpha \beta \in S(q, r)$, we have

$$
q=|X \backslash X \alpha \beta|=|X \backslash \operatorname{dom} \alpha|=g(\alpha) \leq r .
$$

Now we suppose that $q \leq r$ and $g(\alpha)=q$. From the above notation of $\alpha$, since $q=d(\alpha)=\left|X \backslash\left\{x_{i}\right\}\right|$ and $q=g(\alpha)=\left|X \backslash\left\{a_{i}\right\}\right|$, we have

$$
\beta=\left(\begin{array}{c}
x_{i} \\
a_{i}
\end{array}\right) \in S(q, r)
$$

and $\alpha=\alpha \beta \alpha$. Therefore $\alpha$ is regular in $S(q, r)$.

In view of [3] Corollary 1 and Theorem 3, we have proved the following result.

Corollary 1. Suppose that $|X| \geq q \geq \aleph_{0},|X| \geq r \geq \aleph_{0}$ and $q \leq r$. Then

$$
R(q)=\{\alpha \in I(X): d(\alpha)=q=g(\alpha)\}
$$

is the largest regular subsemigroup of $S(q, r)$ and it is also an inverse semigroup.

\section{Green's Relations}

The concept of Green's relations on a semigroup was first studied by J.A. Green in 1951 and they have played a fundamental role in a development of semigroup theory. In this paper, the knowledge and the notations about Green's relations, is based on [2] Chapter II.

In [3] Section 4, the authors described the Green's relations on $P S(q)$. Here, we follow some of their arguments to describe the Green's relations on $S(q, r)$. And, as might be expected, our results are similar to those obtained in [3] Section 4.

Theorem 4. Suppose that $|X| \geq q \geq \aleph_{0}$ and $|X| \geq r \geq \aleph_{0}$. If $\alpha, \beta \in$ $S(q, r)$, then $\alpha=\beta \mu$ for some $\mu \in S(q, r)$ if and only if $\operatorname{dom} \alpha \subseteq \operatorname{dom} \beta$. Hence $\alpha \mathcal{R} \beta$ in $S(q, r)$ if and only if $\operatorname{dom} \alpha=\operatorname{dom} \beta$.

Proof. It is clear that, if $\alpha=\beta \mu$ for some $\mu \in S(q, r)$, then $\operatorname{dom} \alpha \subseteq \operatorname{dom} \beta$. For the converse, if $\operatorname{dom} \alpha \subseteq \operatorname{dom} \beta$, we write

$$
\alpha=\left(\begin{array}{c}
a_{i} \\
x_{i}
\end{array}\right), \beta=\left(\begin{array}{ll}
a_{i} & b_{j} \\
y_{i} & z_{j}
\end{array}\right)
$$


where $\left\{b_{j}\right\}=\operatorname{dom} \beta \backslash \operatorname{dom} \alpha$ and $|J|=|\operatorname{dom} \beta \backslash \operatorname{dom} \alpha| \leq|X \backslash \operatorname{dom} \alpha| \leq r$. Since $d(\alpha)=q$, we write $X \backslash\left\{x_{i}\right\}=A \cup \dot{\cup} B$ where $|A|=q=|B|$, and define

$$
\mu=\left(\begin{array}{cc}
y_{i} & X \backslash X \beta \\
x_{i} & A
\end{array}\right),
$$

where $\mu: X \backslash X \beta \rightarrow A$ is a bijection. Then $\alpha=\beta \mu$, and $\mu \in S(q, r)$ since $d(\mu)=|B|=q$ and $g(\mu)=|J| \leq r$.

To describe the $\mathcal{L}$ relation on $S(q, r)$, we recall from [3] Theorem 8 that, $\alpha \mathcal{L} \beta$ in $P S(q)$ if and only if

$$
(X \alpha=X \beta \text { and } g(\alpha)=g(\beta) \geq q) \text { or } \quad(\alpha=\beta \text { and } g(\alpha)<q) \text {. }
$$

By modifying some arguments in the proof of [3] Theorem 8, we obtain the following result.

Theorem 5. Suppose that $|X| \geq q \geq \aleph_{0}$ and $|X| \geq r \geq \aleph_{0}$. Let $\alpha, \beta \in$ $S(q, r)$. Then $\alpha=\gamma \beta$ for some $\gamma \in S(q, r)$ if and only if $X \alpha \subseteq X \beta$ and

$$
q \leq \max (g(\beta),|X \beta \backslash X \alpha|) \leq \max (g(\alpha), q) .
$$

Hence:

(a) if $q \leq r$, then $\alpha \mathcal{L} \beta$ in $S(q, r)$ if and only if

$$
(X \alpha=X \beta \text { and } g(\alpha)=g(\beta) \geq q) \text { or }(\alpha=\beta \text { and } g(\alpha)<q) \text {; }
$$

(b) if $q>r$, then $\alpha \mathcal{L} \beta$ in $S(q, r)$ if and only if $\alpha=\beta$.

Proof. Suppose that $\alpha, \beta \in S(q, r)$ and $\alpha=\gamma \beta$ for some $\gamma \in S(q, r)$. Then $X \alpha \subseteq X \beta$ and

$$
|\operatorname{dom} \beta \backslash X \gamma|=|X \beta \backslash X \gamma \beta|=|X \beta \backslash X \alpha| \leq|X \backslash X \alpha|=q
$$

Then

$$
\begin{aligned}
q=|X \backslash X \gamma| & =|(X \backslash X \gamma) \cap(X \backslash \operatorname{dom} \beta)|+|\operatorname{dom} \beta \backslash X \gamma| \\
& \leq g(\beta)+|X \beta \backslash X \alpha|=\max (g(\beta),|X \beta \backslash X \alpha|)
\end{aligned}
$$

It remains to show $\max (g(\beta),|X \beta \backslash X \alpha|) \leq \max (g(\alpha), q)$. Since $\alpha=\gamma \beta$, we have

$$
\operatorname{dom} \alpha=\operatorname{dom} \gamma \beta=(X \gamma \cap \operatorname{dom} \beta) \gamma^{-1}
$$


and this follows that $(X \gamma \backslash \operatorname{dom} \beta) \gamma^{-1} \subseteq X \backslash \operatorname{dom} \alpha$, and so $|X \gamma \backslash \operatorname{dom} \beta| \leq$ $|X \backslash \operatorname{dom} \alpha|$. Therefore,

$$
\begin{aligned}
g(\beta)=|X \backslash \operatorname{dom} \beta| & =|X \gamma \backslash \operatorname{dom} \beta|+|(X \backslash X \gamma) \cap(X \backslash \operatorname{dom} \beta)| \\
& \leq|X \backslash \operatorname{dom} \alpha|+q=\max (g(\alpha), q) .
\end{aligned}
$$

We also see that $|X \beta \backslash X \alpha| \leq|X \backslash X \alpha|=q$. Hence, $\max (g(\beta),|X \beta \backslash X \alpha|) \leq$ $\max (g(\alpha), q)$ as required.

For the converse, we suppose the conditions hold. Since $X \alpha \subseteq X \beta$, we may write

$$
\alpha=\left(\begin{array}{c}
a_{i} \\
b_{i}
\end{array}\right) \text { and } \beta=\left(\begin{array}{cc}
c_{i} & c_{j} \\
b_{i} & b_{j}
\end{array}\right)
$$

where $\left|\left\{c_{j}\right\}\right|=|X \beta \backslash X \alpha| \leq q$. We also define

$$
\gamma=\left(\begin{array}{c}
a_{i} \\
c_{i}
\end{array}\right)
$$

then $g(\gamma)=g(\alpha) \leq r$ and $\alpha=\gamma \beta$. It remains to show $d(\gamma)=q$. If $g(\alpha)<q$, then $\max (g(\beta),|X \beta \backslash X \alpha|)=q$ by (1). Also, if $\max (g(\beta),|X \beta \backslash X \alpha|)=\mid X \beta \backslash$ $X \alpha \mid$, then

$$
q \leq \max (g(\beta),|X \beta \backslash X \alpha|)=|X \beta \backslash X \alpha| \leq q
$$

by (1) again, that is, $\max (g(\beta),|X \beta \backslash X \alpha|)=q$. In both cases, we have $d(\gamma)=$ $|J|+g(\beta)=q$, and this follows that $\gamma \in S(q, r)$. Now we suppose that $g(\alpha) \geq q$ and $\max (g(\beta),|X \beta \backslash X \alpha|)=g(\beta)$. Then (1) implies that $q \leq g(\beta) \leq g(\alpha)$. We write $X \backslash \operatorname{dom} \beta=\left\{c_{k}\right\} \dot{\cup}\left\{c_{l}\right\}$ where $|K|=g(\beta),|L|=q$, and choose $\left\{z_{k}\right\} \subseteq X \backslash$ dom $\alpha$ (possible since $g(\beta) \leq g(\alpha)$ ). Then re-define

$$
\gamma=\left(\begin{array}{cc}
a_{i} & z_{k} \\
c_{i} & c_{k}
\end{array}\right)
$$

we have $\alpha=\gamma \beta$ and $\gamma \in S(q, r)$ since $d(\gamma)=\left|\left\{c_{j}\right\} \cup\left\{c_{l}\right\}\right|=q$ and $g(\gamma) \leq$ $g(\alpha) \leq r$.

Now, to see (a), we suppose that $q \leq r$ and let $\alpha, \beta \in S(q, r)$ be such that $\alpha \neq \beta$ and $\alpha \mathcal{L} \beta$. Thus, $\alpha=\gamma \beta$ and $\beta=\mu \alpha$ for some $\gamma, \mu \in S(q, r)$. Then $X \alpha=X \beta$ and so $|X \beta \backslash X \alpha|=0=|X \alpha \backslash X \beta|$. Therefore, $q \leq g(\alpha)=g(\beta)$ by (1). Also, the converse is quite obvious. In particular, when $r<q, S(q, r)$ contains only mappings with gap less than $q$, therefore $\alpha \mathcal{L} \beta$ if and only if $\alpha=\beta$, that is (b) holds.

Since $\mathcal{H}=\mathcal{L} \cap \mathcal{R}$, Theorem 4 and Theorem 5 lead to our next result. 
Corollary 2. Suppose that $|X| \geq q \geq \aleph_{0}$ and $|X| \geq r \geq \aleph_{0}$. If $\alpha, \beta \in$ $S(q, r)$, then $\alpha \mathcal{H} \beta$ in $S(q, r)$ if and only if

$(\operatorname{dom} \alpha=\operatorname{dom} \beta, X \alpha=X \beta$ and $g(\alpha)=g(\beta) \geq q)$ or $(\alpha=\beta$ and $g(\alpha)<q)$.

It quite difficult to describe the $\mathcal{J}$ relation on $S(q, r)$, so we will consider in the following three cases.

Lemma 1. Suppose that $|X| \geq q \geq \aleph_{0},|X| \geq r \geq \aleph_{0}$ and $q<r$. If $\alpha, \beta \in S(q, r)$, then $\beta=\lambda \alpha \mu$ for some $\lambda, \mu \in S(q, r)$ if and only if $g(\alpha) \leq q$ or $g(\beta) \geq g(\alpha)>q$.

Hence, $\alpha \mathcal{J} \beta$ in $S(q, r)$ if and only if

$$
\max (g(\alpha), g(\beta)) \leq q \text { or } g(\alpha)=g(\beta)>q
$$

Proof. Suppose that the conditions hold and let $\beta=\lambda \alpha \mu$ for some $\lambda, \mu \in$ $S(q, r)$. We assume $q<g(\alpha)$. Since

$$
g(\alpha)=|(X \backslash X \lambda) \cap(X \backslash \operatorname{dom} \alpha)|+|X \lambda \backslash \operatorname{dom} \alpha|
$$

where $|(X \backslash X \lambda) \cap(X \backslash \operatorname{dom} \alpha)| \leq q$, we have $|X \lambda \backslash \operatorname{dom} \alpha|=g(\alpha)>q$. Also, since $\operatorname{dom} \beta=(X \lambda \cap \operatorname{dom} \alpha \mu) \lambda^{-1}$, we have $(X \lambda \backslash \operatorname{dom} \alpha \mu) \lambda^{-1} \subseteq X \backslash \operatorname{dom} \beta$. It follows that

$$
\begin{aligned}
q<g(\alpha) & =|X \lambda \backslash \operatorname{dom} \alpha| \leq|X \lambda \backslash \operatorname{dom} \alpha \mu| \\
& =\left|(X \lambda \backslash \operatorname{dom} \alpha \mu) \lambda^{-1}\right| \leq|X \backslash \operatorname{dom} \beta|=g(\beta)
\end{aligned}
$$

For the converse, since $d(\alpha)=q<r \leq|X|$, we have $r(\alpha)=|X|$. Similarly, $r(\beta)=|X|$. Then we can write

$$
\alpha=\left(\begin{array}{l}
a_{i} \\
b_{i}
\end{array}\right) \text { and } \beta=\left(\begin{array}{c}
c_{i} \\
d_{i}
\end{array}\right),
$$

where $|I|=|X|$. If $g(\alpha) \leq q$, then we write $\left\{a_{i}\right\}=\left\{x_{i}\right\} \dot{\cup}\left\{x_{j}\right\}$ where $|J|=q$. Then $X \alpha=\left\{x_{i} \alpha\right\} \dot{\cup}\left\{x_{j} \alpha\right\}$. Next, we define

$$
\lambda=\left(\begin{array}{c}
c_{i} \\
x_{i}
\end{array}\right) \text { and } \mu=\left(\begin{array}{c}
x_{i} \alpha \\
d_{i}
\end{array}\right),
$$

then $\beta=\lambda \alpha \mu$. We can see that $g(\lambda)=g(\beta) \leq r$ and $d(\lambda)=\left|\left\{x_{j}\right\}\right|+g(\alpha)=q$, that is $\lambda \in S(q, r)$. Moreover, $g(\mu)=\left|\left\{x_{j} \alpha\right\}\right|+d(\alpha)=q<r$ and $d(\mu)=d(\beta)=$ 
$q$, this means that $\mu \in S(q, r)$. On the other hand, if $q<g(\alpha) \leq g(\beta)$, then we write

$$
X \backslash \operatorname{dom} \alpha=\left\{m_{k}\right\} \dot{\cup} A \text { and } X \backslash \operatorname{dom} \beta=\left\{n_{k}\right\} \dot{\cup} B
$$

where $|K|=g(\alpha),|A|=q$ and $|B|=g(\beta)$. Then re-define

$$
\lambda=\left(\begin{array}{cc}
c_{i} & n_{k} \\
a_{i} & m_{k}
\end{array}\right) \text { and } \mu=\left(\begin{array}{c}
b_{i} \\
d_{i}
\end{array}\right)
$$

we have $\beta=\lambda \alpha \mu$ and $g(\lambda)=g(\beta) \leq r, d(\lambda)=|A|=q, g(\mu)=d(\alpha)=q<r$ and $d(\mu)=d(\beta)=q$, that is, $\lambda, \mu \in S(q, r)$.

Lemma 2. Suppose that $|X| \geq q \geq \aleph_{0},|X| \geq r \geq \aleph_{0}$ and $q=r$. If $\alpha, \beta \in S(q, r)$, then $\beta=\lambda \alpha \mu$ for some $\lambda, \mu \in S(q, r)$ if and only if $r(\beta) \leq r(\alpha)$.

Hence,

$$
\alpha \mathcal{J} \beta \text { in } S(q, r) \text { if and only if } r(\alpha)=r(\beta) \text {. }
$$

Proof. Suppose that the conditions hold. If $\beta=\lambda \alpha \mu$, then it is clear that $r(\beta) \leq r(\alpha)$. Conversely, suppose that $r(\beta) \leq r(\alpha)$. Then we write

$$
\alpha=\left(\begin{array}{c}
a_{i} \\
b_{i}
\end{array}\right) \text { and } \beta=\left(\begin{array}{c}
c_{j} \\
d_{j}
\end{array}\right) \text { where }|J| \leq|I|
$$

and write $\left\{a_{i}\right\}=\left\{x_{j}\right\} \dot{\cup}\left\{x_{k}\right\}$. Define

$$
\lambda=\left(\begin{array}{c}
c_{j} \\
x_{j}
\end{array}\right) \text { and } \mu=\left(\begin{array}{c}
x_{j} \alpha \\
d_{j}
\end{array}\right)
$$

then $\beta=\lambda \alpha \mu$ and it is clear that $g(\lambda)=g(\beta) \leq r, d(\mu)=d(\beta)=q$. We also see that

$$
d(\lambda)=\left|\left\{x_{k}\right\}\right|+g(\alpha) \text { and } g(\mu)=\left|\left\{x_{k} \alpha\right\}\right|+d(\alpha) .
$$

If $|X|>q$, then $r(\alpha)=r(\beta)=|X|$, that is, $|I|=|J|=|X|$. Thus, when writing $\left\{a_{i}\right\}=\left\{x_{j}\right\} \dot{\cup}\left\{x_{k}\right\}$, we can assume $\left|\left\{x_{k}\right\}\right|=q$. It follows that $d(\lambda)=q$ and $g(\mu)=q=r$, that is, $\lambda, \mu \in S(q, r)$. On the other hand, if $|X|=q$, then $|X|=q=r$ and thus $g(\mu)=r$. In this case, if $g(\alpha)=|X|$, then $d(\lambda)=|X|=q$, otherwise, if $g(\alpha)<|X|$, then $|I|=|X|$. It follows that, when writing $\left\{a_{i}\right\}=\left\{x_{j}\right\} \dot{\cup}\left\{x_{k}\right\}$, we can assume $\left|\left\{x_{k}\right\}\right|=|X|$. Thus $d(\lambda)=|X|=q$ and therefore $\lambda, \mu \in S(q, r)$.

Lemma 3. Suppose that $|X| \geq q \geq \aleph_{0},|X| \geq r \geq \aleph_{0}$ and $q>r$. Then the $\mathcal{J}$ relation is the universal relation on $S(q, r)$. 
Proof. Suppose that the conditions hold and let $\alpha, \beta \in S(q, r)$. Since $g(\alpha) \leq r<q \leq|X|$, we have $r(\alpha)=|X|$, similarly, $r(\beta)=|X|$. We write

$$
\alpha=\left(\begin{array}{c}
a_{i} \\
b_{i}
\end{array}\right) \text { and } \beta=\left(\begin{array}{c}
c_{i} \\
d_{i}
\end{array}\right) \text {, where }|I|=|X| \text {. }
$$

We also write

$$
\left\{a_{i}\right\}=\left\{x_{i}\right\} \dot{\cup}\left\{x_{j}\right\}, X \backslash X \alpha=A \dot{\cup} B \text { and } X \backslash X \beta=C \dot{\cup} D,
$$

where $|J|=q,|A|=|C|=|D|=q$ and $|B|=r$. Then $X \alpha=\left\{x_{i} \alpha\right\} \dot{\cup}\left\{x_{j} \alpha\right\}$. Define

$$
\lambda=\left(\begin{array}{c}
c_{i} \\
x_{i}
\end{array}\right), \text { and } \mu=\left(\begin{array}{cc}
x_{i} \alpha & \left\{x_{j} \alpha\right\} \cup A \\
d_{i} & C
\end{array}\right),
$$

where $\mu:\left\{x_{j} \alpha\right\} \cup A \rightarrow C$ is a bijection. Then $\beta=\lambda \alpha \mu$, and $g(\lambda)=g(\beta) \leq r$, $d(\lambda)=\left|\left\{x_{j}\right\}\right|+g(\alpha)=q, g(\mu)=|B|=r$ and $d(\mu)=|D|=q$, that is $\lambda, \mu \in S(q, r)$. Similarly, we can define $\lambda^{\prime}, \mu^{\prime} \in S(q, r)$ such that $\alpha=\lambda^{\prime} \beta \mu^{\prime}$. Therefore, $\alpha \mathcal{J} \beta$ and hence $\mathcal{J}=S(q, r) \times S(q, r)$.

Recall from [3] Theorem 9 that, if $\alpha, \beta \in P S(q)$, then $\alpha \mathcal{J} \beta$ in $P S(q)$ if and only if

$$
[\max (g(\alpha), g(\beta)) \leq q \text { and } r(\alpha)=r(\beta)] \text { or }[g(\alpha)=g(\beta)>q]
$$

In what follows, by using Lemma 1, Lemma 2 and Lemma 3, we reach the same result for $\alpha, \beta \in S(q, r)$.

Theorem 6. Suppose that $|X| \geq q \geq \aleph_{0}$ and $|X| \geq r \geq \aleph_{0}$. If $\alpha, \beta \in$ $S(q, r)$, then $\alpha \mathcal{J} \beta$ in $S(q, r)$ if and only if

$$
[\max (g(\alpha), g(\beta)) \leq q \text { and } r(\alpha)=r(\beta)] \text { or }[g(\alpha)=g(\beta)>q] .
$$

Proof. Suppose that the conditions hold and let $\alpha, \beta \in S(q, r)$. If $q<r$, then $r(\alpha)=|X|=r(\beta)$ since $d(\alpha)=d(\beta)=q<r \leq|X|$. Thus (2) and (4) are equivalent. Next, if $q=r$, then $g(\alpha) \leq r=q$ and similarly, $g(\beta) \leq q$. Therefore $\max (g(\alpha), g(\beta)) \leq q$ and so (3) and (4) are equivalent. Finally, if $q>r$, then $|\operatorname{dom} \alpha|=|X|$ since $g(\alpha) \leq r<q \leq|X|$. Then $r(\alpha)=|X|$ since $\alpha$ is injective. Similarly, $r(\beta)=|X|$ and this follows that $r(\alpha)=r(\beta)$. Thus, the condition (4) is simply the universal relation on $S(q, r)$. Therefore, in each case $\alpha \mathcal{J} \beta$ in $S(q, r)$ if and only if $\alpha$ and $\beta$ satisfy (4). 
Theorem 7. Suppose that $|X| \geq q \geq \aleph_{0}$ and $|X| \geq r \geq \aleph_{0}$. Let $\alpha, \beta \in$ $S(q, r)$. Then $\alpha \mathcal{D} \beta$ in $S(q, r)$ if and only if

$$
(r(\alpha)=r(\beta) \text { and } g(\alpha)=g(\beta) \geq q) \text { or }(\operatorname{dom} \alpha=\operatorname{dom} \beta \text { and } g(\alpha)<q) \text {. }
$$

In particular, if $q>r$, then $\alpha \mathcal{D} \beta$ in $S(q, r)$ if and only if $\operatorname{dom} \alpha=\operatorname{dom} \beta$.

Proof. Suppose that the conditions hold. If $\alpha \mathcal{D} \beta$ in $S(q, r)$, then $\alpha \mathcal{L} \gamma \mathcal{R} \beta$ for some $\gamma \in S(q, r)$. If $\alpha=\gamma$, then $\alpha \mathcal{R} \beta$, that is, $\operatorname{dom} \alpha=\operatorname{dom} \beta$ by Theorem 4 , and this leads us to (5). Otherwise, if $\alpha \neq \gamma$, then Theorem 4 and Theorem 5 imply that $X \alpha=X \gamma, g(\alpha)=g(\gamma) \geq q$ and $\operatorname{dom} \gamma=\operatorname{dom} \beta$. Therefore $q \leq g(\alpha)=g(\beta)$ and $r(\alpha)=r(\beta)$, that is, (5) holds. Conversely, since $\mathcal{R} \subseteq \mathcal{D}$, if $\operatorname{dom} \alpha=\operatorname{dom} \beta$, then $\alpha \mathcal{R} \beta$ by Theorem 4 and so $\alpha \mathcal{D} \beta$. For the case that $r(\alpha)=r(\beta)$ and $g(\alpha)=g(\beta) \geq q$, we write

$$
\alpha=\left(\begin{array}{c}
a_{i} \\
b_{i}
\end{array}\right), \beta=\left(\begin{array}{c}
c_{i} \\
d_{i}
\end{array}\right) \text { and let } \gamma=\left(\begin{array}{c}
c_{i} \\
b_{i}
\end{array}\right) .
$$

Then $g(\gamma)=g(\beta) \leq r$ and $d(\gamma)=d(\alpha)=q$, that is, $\gamma \in S(q, r)$. Moreover, $\alpha \mathcal{L} \gamma \mathcal{R} \beta$ by Theorem 4 and Theorem 5, therefore $\alpha \mathcal{D} \beta$ as required. In particular, if $q>r$, then $g(\alpha) \leq r<q$ for all $\alpha \in S(q, r)$. Thus, by (5), $\alpha \mathcal{D} \beta$ if and only if $\operatorname{dom} \alpha=\operatorname{dom} \beta$.

\section{Two-Sided Ideals}

In what follows, we first recall that a semigroup $S$ without zero is called simple if it has no proper ideals and it is known that $S$ is simple if and only if $\mathcal{J}=S \times S$ (see [2] Chapter III). Also, let $u$ be a cardinal number, the successor of $u$ is denoted by $u^{\prime}$, where

$$
u^{\prime}=\min \{v: v>u\}
$$

Note that $u^{\prime}$ always exists since the cardinals are well-ordered, and when $u$ is finite we have $u^{\prime}=u+1$.

Theorem 8. Suppose that $|X| \geq q \geq \aleph_{0}$ and $|X| \geq r \geq \aleph_{0}$. Then the following statements hold:

(a) if $q>r$, then $S(q, r)$ has no proper ideals;

(b) if $q=r$, then $S(q, r)$ has a proper ideal precisely when $|X|=q$ and, in this case, all ideals of $S(q, r)$ are of the form

$$
V_{t}=\{\alpha \in S(q, r): r(\alpha)<t\}
$$


where $1 \leq t \leq|X|^{\prime}$. Moreover, $V_{t}$ is principal precisely when $t=s^{\prime}$ where $0 \leq s \leq|X|$

(c) if $q<r$, then the proper ideals of $S(q, r)$ are precisely the sets:

$$
U_{t}=\{\alpha \in S(q, r): g(\alpha) \geq t\}
$$

where $q<t \leq r$. Moreover, each $U_{t}$ is a principal ideal.

Proof. Suppose that the conditions hold. Here, for convenience we write $S$ in place of $S(q, r)$. If $q>r$, then Lemma 3 implies that $\mathcal{J}=S \times S$ and so $S(q, r)$ is simple. It follows that (a) holds.

For (b), we suppose that $q=r$. In this case, if $|X|>q$, then every element in $S$ has the same rank $|X|$. Then, by Lemma $2, \mathcal{J}=S \times S$ and thus $S$ has no proper ideals. We now suppose $|X|=q$. It follows that $|X|=q=r$ and thus $S=P S(q)$. Hence, all ideals of $S$ are exactly the same as those ideals of $P S(q)$. Therefore, we have completed this case by [ps] Theorem 14 .

Finally, to prove (c), suppose that $q<r$ and let $t$ be a cardinal such that $q<t \leq r$. To show $U_{t}$ is an ideal. Let $\alpha \in U_{t}$ and $\beta \in S$. Since $\operatorname{dom} \alpha \beta \subseteq \operatorname{dom} \alpha$, we have $t \leq g(\alpha) \leq g(\alpha \beta)$. Therefore $U_{t}$ is a right ideal. Also, since

$$
X \backslash \operatorname{dom} \alpha=[X \beta \cap(X \backslash \operatorname{dom} \alpha)] \dot{\cup}[(X \backslash X \beta) \cap(X \backslash \operatorname{dom} \alpha)]
$$

where $g(\alpha) \geq t>q$ and the last term has cardinality at most $q$ (since $|X \backslash X \beta|=$ $q)$, we have $|X \beta \cap(X \backslash \operatorname{dom} \alpha)| \geq t$. This means that

$$
t \leq\left|[X \beta \cap(X \backslash \operatorname{dom} \alpha)] \beta^{-1}\right|=|\operatorname{dom} \beta \backslash \operatorname{dom} \beta \alpha| .
$$

Thus, the equation

$$
X \backslash \operatorname{dom} \beta \alpha=(X \backslash \operatorname{dom} \beta) \dot{\cup}(\operatorname{dom} \beta \backslash \operatorname{dom} \beta \alpha)
$$

implies that $g(\beta \alpha) \geq t$, that is, $U_{t}$ is a left ideal. For the converse, let $I$ be a proper ideal of $S$ and choose $\alpha$ with the least gap in $I$, say $g(\alpha)=t$, then $t \leq r$ and $I \subseteq U_{t}$. We claim that $q<t \leq r$. Suppose not, that is $t \leq q$ and we also note that, since $q<r$, we have that every element in $S$ has the same rank $|X|$. Then, let $\beta \in S$ and write

$$
\alpha=\left(\begin{array}{l}
a_{i} \\
b_{i}
\end{array}\right), \text { then } \beta=\left(\begin{array}{l}
c_{i} \\
d_{i}
\end{array}\right),
$$

where $|I|=|X|$. We also write

$$
\left\{a_{i}\right\}=\left\{x_{i}\right\} \dot{\cup}\left\{x_{j}\right\}, X \backslash X \alpha=A \dot{\cup} B, X \backslash X \beta=C \dot{\cup} D,
$$


where $|J|=q,|A|=|C|=|D|=q$ and $|B|=t$. Then $X \alpha=\left\{x_{i} \alpha\right\} \dot{\cup}\left\{x_{j} \alpha\right\}$. Define

$$
\lambda=\left(\begin{array}{c}
c_{i} \\
x_{i}
\end{array}\right) \text { and } \mu=\left(\begin{array}{cc}
x_{i} \alpha & \left\{x_{j} \alpha\right\} \cup A \\
d_{i} & C
\end{array}\right)
$$

where $\mu:\left\{x_{j} \alpha\right\} \cup A \rightarrow C$ is a bijection. Then $\beta=\lambda \alpha \mu$, and $g(\lambda)=g(\beta) \leq r$, $d(\lambda)=\left|\left\{x_{j}\right\}\right|+g(\alpha)=q+t=q, g(\mu)=|B|=t \leq r$ and $d(\mu)=|D|=q$, that is $\lambda, \mu \in S$. Therefore $S=S . \alpha . S \subseteq I$ (since $I$ is an ideal), a contradiction. Hence $q<t \leq r$. Also, for each $\gamma \in U_{t}$, we have $g(\gamma) \geq t=g(\alpha)>q$, thus, Lemma 1 implies that $\gamma=\delta \alpha \epsilon \in I$ for some $\delta, \epsilon \in S$. Therefore $U_{t}=I$. Finally, for each $q<t \leq r$, choose $\alpha \in U_{t}$ with gap $t$. Then, for each $\beta \in U_{t}$, $g(\beta) \geq g(\alpha)=t>q$. Again, by Lemma 1, we have that $\beta=\lambda \alpha \mu$ for some $\lambda, \mu \in S$. Therefore $U_{t} \subseteq S^{1} . \alpha . S^{1} \subseteq U_{t}$ since $U_{t}$ is an ideal, and so $U_{t}=S^{1} . \alpha . S^{1}$, that is, $U_{t}$ is principal.

\section{Acknowledgments}

The author thanks the Faculty of Science and Technology, Chiangmai Rajabhat University, Thailand, for the financial support during the preparation of this research.

\section{References}

[1] A.H. Clifford, G.B. Preston, The algebraic theory of semigroups, Mathematical Surveys, 7, No-s: 1 and 2, American Mathematical Society, Providence, RI, 1961 and 1967.

[2] J.M. Howie, An Introduction to Semigroup Theory, Academic Press, London (1976).

[3] Fernanda A. Pinto, R.P. Sullivan, Baer-Levi semigroups of partial transformations, Bull. Austral. Math. Soc., 69, No. 1 (2004), 87-106.

[4] B. Singha, J. Sanwong, On maximal subsemigroups of partial Baer-Levi semigroups, Int. J. Math. Math. Sci. (2011), 14 pp., Art. ID 489674. 
